\title{
Th1/Th2 PB balance and CD200 expression of patients with active severe alopecia areata
}

\author{
XINHUA MA, SHISHENG CHEN, WANWAN JIN and YU GAO \\ Department of Dermatology, The Second Affiliated Hospital and Yuying Children's Hospital \\ of Wenzhou Medical University, Wenzhou, Zhejiang 325027, P.R. China
}

Received May 27, 2016; Accepted January 25, 2017

DOI: $10.3892 /$ etm.2017.4312

\begin{abstract}
Th1/Th2 peripheral blood balance and CD200 expression in patients with severe alopecia areata (SAA) in the active stage were investigated. Fifty patients with active SAA, 50 patients with stable SAA and 50 healthy controls were continuously selected and expression of Th1/Th2 of peripheral $\mathrm{T}$ lymphocytes, and peripheral B lymphocytes was detected by flow cytometry; RT-PCR was used to detect the expression of the PBMC CD200 mRNA and the expression of CD200 in hair follicles of alopecia area was detected by immunohistochemically staining; ELISA was used to detect expression levels of serum LFN- $\gamma$ and serum interleukin (IL)-10. The expression of CD200 in patients with alopecia areata in active phase on $\mathrm{CD}^{+} \mathrm{T}$ lymphocytes and $\mathrm{CD} 19^{+} \mathrm{B}$ lymphocytes was significantly lower $(\mathrm{P}<0.05)$ than those in stable phase and of the control group. CD200 expression in patients with alopecia areata in stable phase on $\mathrm{T}$ lymphocytes was greatly lower than that of the control group $(\mathrm{P}<0.05)$. However, the comparison of expression of CD200 in patients with alopecia areata in stable phase on B lymphocytes with the control group were statistically non-significant. The level of the expression of CD200 mRNA in active phase was obviously lower than those of the other two groups and the difference was statistically significant $(\mathrm{P}<0.05)$; the moderate positive and strong positive percentage of CD200 in the active phase was significantly lower than those of the other two groups. Positive expression rate of CK15 among the three groups were compared with each other; the differences had no statistical significance. The level of LFN- $\gamma$ in the active phase had obviously increased while the IL-10 level decreased significantly $(\mathrm{P}<0.05)$. In conclusion, the level of expression of CD200 on peripheral blood and hair
\end{abstract}

Correspondence to: Dr Yu Gao, Department of Dermatology, The Second Affiliated Hospital and Yuying Children's Hospital of Wenzhou Medical University, 109 Xueyuan Western Road, Wenzhou, Zhejiang 325027, P.R. China

E-mail: yu_gao_1212@163.com

Key words: severe alopecia areata, Th1/Th2, CD200, flow cytometry, RT-PCR, immunohistochemical staining, enzyme-linked immunosorbent assay follicle outer root sheath of patients with SAA was decreased. This may be associated with the imbalance of the Th1/Th2 equilibrium.

\section{Introduction}

Alopecia areata, as a common alopecia disease, is considered as a hair follicle-specific Th1 cell-mediated autoimmune disease (1). Some studies suggested that the hair follicle was an immune privileged organ, but it is still not clear how the patients with alopecia areata generate the autoimmune reaction mechanism in terms of hair follicle (2). CD200 is an immune regulatory molecule discovered recently (3). CD200 can be expressed on a variety of cell surfaces, including hair follicle. It is believed that it has great effect on protecting immune system (4). This study focused on the CD200 expression of severe alopecia areata (SAA) patients, and peripheral blood and hair follicle was analyzed for its role in immune tolerance, to explore the pathogenesis of alopecia areata and potential therapeutic targets.

\section{Patients and methods}

Patient information. The study obtained the approval of the Ethics Committee of the Second Affiliated Hospital and Yuying Children's Hospital of Wenzhou Medical University and written informed consent was obtained from patients, or their family members. We continuously selected 50 cases of patients with SAA in active phase and 50 cases in stable phase who were admitted and diagnosed in the Second Affiliated Hospital and Yuying Children's Hospital of Wenzhou Medical University from January 2015 to January 2016. The diagnosis criteria of severe alopecia areata referred to inflammation manifestations put forward in 2004 by Olsen et al (5), including alopecia area $>50 \%$ area of the scalp, normal state of skin lesions and no erythema scales. Active phase was characterized by continued loss of body hair in the preceding 1 month, while stable phase was characterized by no continued loss of body hair. Both phases were proven by pathologic biopsy. In the preceding 1 month, patients who applied corticosteroid and other drugs for promoting hair growth as well as those with autoimmune diseases, who were pregnant or at lactation, with combined inherited metabolic diseases, and with other severe visceral organ dysfunction, poor compliance, involved in other studies, 
and voluntary withdrawal during the study, were excluded. Moreover, 50 healthy persons of the same phase were selected as the control group.

There were 32 males and 18 females in active phase; aged from 20 to 60 years with an average age of $41.2 \pm 13.5$ years; the course of the disease was from 1 month to 5 years with median time of 10.6 months; alopecia area accounted for 50 to $85 \%$ of the area of the scalp with an average of $67.8 \pm 10.6 \%$. There were 30 males and 20 females in stable phase, aged from 18 to 63 years with an average age of $40.9 \pm 14.4$ years; course of the disease was from 1.5 months to 4.5 years with median time of 10.3 months; alopecia area accounted for 55 to $80 \%$ of the area of the scalp with an average of $65.2 \pm 13.2 \%$. There were 28 males and 22 females in the control group; aged from 22 to 69 years with an average age of $43.5 \pm 16.1$ years. Comparison of the gender and age among the three groups showed no statistically significant difference; the course of disease and alopecia area of patients with SAA in active and stable phase were compared, and the difference was not statistically significant.

Research method. Flow cytometry was adopted to detect the expression of Th1/Th2 of peripheral blood $\mathrm{T}$ and B lymphocytes; RT-PCR was used to detect the expression of mononuclear cell CD200 mRNA; immunohistochemical staining to detect the expression of CD200 in hair follicles in the alopecia area; enzyme-linked immunosorbent assay (ELISA) to detect the expression level of serum interferon IFN- $\gamma$ and interleukin- 10 .

Flow cytometry detection. Mouse monoclonal CD3 antibody labeled with PerCP (dilution, 1:100; cat. no. sc-18871), mouse monoclonal CD19 antibody labeled with FITC (dilution, 1:100; cat. no. sc-69736), mouse monoclonal CD200 antibody labeled with PE (dilution, 1:100; cat. no. sc-71761) were from Santa Cruz Biotechnology, Inc. (Santa Cruz, CA, USA). Igg homotype contrast labeled by corresponding fluorescence, and BD FACSAria sorting flow cytometry (BD Biosciences, Franklin Lakes, NJ, USA).

Main steps: 5-ml anticoagulant venous blood was drawn and detected immediately. Fifty microliters was used for flow cytometry detection (tested within $48 \mathrm{~h}$ ), and the remaining $4 \mathrm{ml}$ was used to separate PBMC immediately to detect the content of CD200 mRNA. Ten microliters of CD200 antibody, $10 \mu 1$ of CD3, $10 \mu 1$ of CD19 labeled with different fluorescence and $10 \mu 1$ of the homotype contrast were put into test tubes with coding sequences; $50 \mu \mathrm{l}$ anticoagulant peripheral blood of the three groups was added, and then incubated for $15 \mathrm{~min}$ in the dark, at room temperature; 1-ml of hemolysin was added and then reacted for $15 \mathrm{~min}$ in the dark, at room temperature; followed byphosphate-buffered saline (PBS) washing, centrifuging, and the washing liquid was discarded; $500 \mu \mathrm{l}$ of PBS was added to cell suspension. Flow cytometry and CellQuest software version 3.3 (BD Biosciences, San Jose, CA, USA) were used to conduct detection. The homotype contrast IgG staining cells labeled with corresponding fluorescence were considered as negative control, and lymphocytes were considered as the evaluation border. Ten thousand cells were collected inside the border and then CD200 expression on the surfaces of $\mathrm{T}$ and $\mathrm{B}$ lymphocytes were detected.
$R T-P C R$. Main reagents and equipment: Lymphocyte separation liquid (Nanjing Kaiji Biology Development Co., Ltd., Nanjing, China), TRIzol (Invitrogen, Carslbad, CA, USA), RT-PCR kit (Fermentas, Vilnius, Lithuania), ultraviolet spectrometer (Shimadzu Corp., Kyoto, Japan), nucleic acid electrophoresis apparatus (Beijing 6-1 Instrument Factory, Beijing, China), and gel imaging instrument (Bio-Rad Laboratories, Inc., Hercules, CA, USA).

Main steps: Density gradient centrifugation was used to separate PBMC. TRIzol was used to extract total RNA. Ultraviolet spectrophotometry was used to test concentration and purity. Reverse transcription kit was used to synthesize cDNA, and CD200 and internal reference $\beta$-actin primers were synthesized by Genscript (Nanjing) Co., Ltd. (Nanjing, China): CD200 forward, 5'-AGCAAAAGAGGTGGGAGC-3' and reverse, 5'-GTAACATCAACAGAAAGGCA-3', 415 bp; $\beta$-actin forward, 5'-CGGGAAATCGTGCGTGAC-3' and reverse, 5'-TGGAAGGTGGACAGCGAGG-3', 443 bp. The reaction system included 10X Taq buffer solution $5 \mu 1$, dNTPs $(10 \mathrm{mM}) 1 \mu 1$, upstream and downstream primers $(10 \mathrm{mM})$ $2 \mu 1,2 \mu 1$ cDNA template, $0.5 \mu 1$ Taq DNA polymerase, $4 \mu 1$ $\mathrm{MgCl}_{2}$, adding sterilization double distilled water to total volume $50 \mu \mathrm{l}$. Reaction conditions were $95^{\circ} \mathrm{C}$ for $5 \mathrm{~min}, 95^{\circ} \mathrm{C}$ for $40 \mathrm{sec}, 50^{\circ} \mathrm{C}$ for $30 \mathrm{sec}$ and $72^{\circ} \mathrm{C}$, for $35 \mathrm{sec}, 33$ cycles, $72^{\circ} \mathrm{C}$ for $10 \mathrm{~min}$. After the reaction, $5-\mu 1$ reaction solution was taken to carry out $1 \%$ agarose gel electrophoresis. Positions and brightness of the product strip were observed under UV lamp and gel imaging system was used for semi-quantitative analysis on target genes and internal reference amplification products, the results are presented with the $2^{-\Delta \Delta C t}$ method.

Immunohistochemical staining. Mouse monoclonal keratin 15 (CK15) antibody (dilution, 1:200; cat. no. sc-53254) and CD200 antibody (dilution, 1:100; cat. no. sc-71760) were from Santa Cruz Biotechnology, Inc. SP super sensitive immunohistochemical kit (R\&D Systems, Minneapolis, MN, USA), Q500MC Leica Image Analysis system (Leica Microsystems GmbH, Wetzlar, Germany) and Zeiss Axio Imager Ml microscope (Carl Zeiss, Jena, Germany).

Main steps: Conventional fixation, embedding, continuous slicing (4- $\mu$ l thick), dewaxing to water, antigen repairing, PBS washing, normal goat serum blocking at $37^{\circ} \mathrm{C}$ and incubated for $10 \mathrm{~min}$, no washing. The appropriate proportion was added of primary antibody working fluid (CD200 1:100 and CK15 1:200), at $4^{\circ} \mathrm{C}$ overnight, re-warming $20 \mathrm{~min}$, washed with PBS for 3 min, 3 times, PBS replaced primary antibody as negative control and the second antibody labeled with biotin (dilution, 1:500; cat. no. sc-73451; Santa Cruz Biotechnology, Inc.) was added, incubated at $37^{\circ} \mathrm{C}$ for $10 \mathrm{~min}$, washed with PBS for 3 min, 3 times, chain enzyme avidin labeled by horseradish peroxidase wa added, incubated at $37^{\circ} \mathrm{C}$ for $10 \mathrm{~min}$, washed with PBS for 3 min, 3 times, DAB coloration, then hematoxylin to stain again, alcohol to hydrate, and neutral gum was used for mounting. The staining was observed under an optical microscope. According to the Shimizu method, the results of staining were interpreted as: Brown granules in the CD200- positive cytomembrane and the CK15-positive cytoplasm. The positive cells were mainly located in the outer root sheath of the hair follicle and the basal layer of epidermis. Five hair follicles were selected randomly at 40 magnification and 

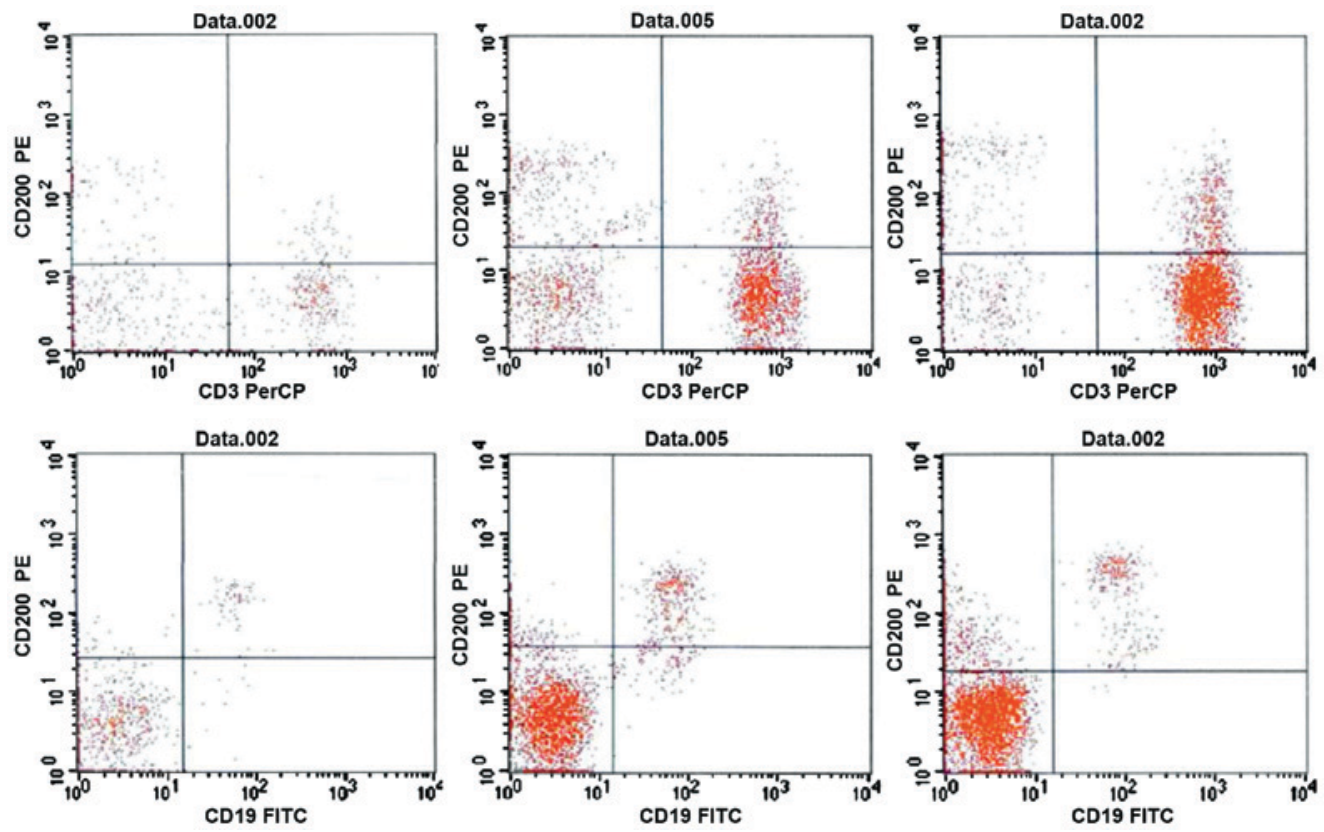

Figure 1. Result of flow cytometry detection. The upper row shows the expression of CD200 on T lymphocytes, the bottom row, on B lymphocytes; the left was in active phase, the middle, in stable phase, the right, the control group.

Table I. Result of flow cytometry detection (\%).

\begin{tabular}{lrc}
\hline Groups & $\mathrm{CD}^{+} \mathrm{T}$ & $\mathrm{CD} 19^{+} \mathrm{B}$ \\
\hline Group in active phase & $6.8 \pm 2.9$ & $63.6 \pm 7.5$ \\
Group in stable phase & $8.2 \pm 3.3$ & $74.2 \pm 7.6$ \\
Control group & $13.5 \pm 4.2$ & $74.9 \pm 7.4$ \\
F-value & 10.326 & 7.524 \\
P-value & $<0.001$ & 0.013 \\
\hline
\end{tabular}

then the number of positive cells was counted in each of the 100 nuclear cells in the field of view. We graded according to the percentage of positive cells. The $<5,5-25,26-50$ and $>51 \%$ were represented by $0-3$, respectively; and positive cells of color intensity as: no-color, 0 , light yellow 1 , brown yellow 2 and brown 3 . The sum of the two scores was the integral of the degree of color: $0-1$ negative (-), 2 points for weakly positive $(+), 3$ points moderately positive $(++)$ and 4 points as strong positive $(+++)$.

ELISA assay. Main reagents and instruments: IFN- $\gamma$, IL-10 kits (Sigma-Aldrich, St. Louis, MO, USA). Well scan MK2 enzyme-linked immune detector (Olympus, Tokyo, Japan), $\mathrm{CO}_{2}$ incubator, transfer decolorization table (Shanghai Jing Hong Experimental Equipment Co., Ltd., Shanghai, China). Experimental procedures were conducted according to the kit instructions.

Statistical analysis. SPSS 19.0 software (IBM, Armonk, NY, USA) was used for input and analysis, quantitative data were expressed by the mean \pm standard deviation, comparison among groups was analyzed by single factor ANOVA, pairwise comparison used LSD method, qualitative data were expressed by the number of cases or a percentage, comparison

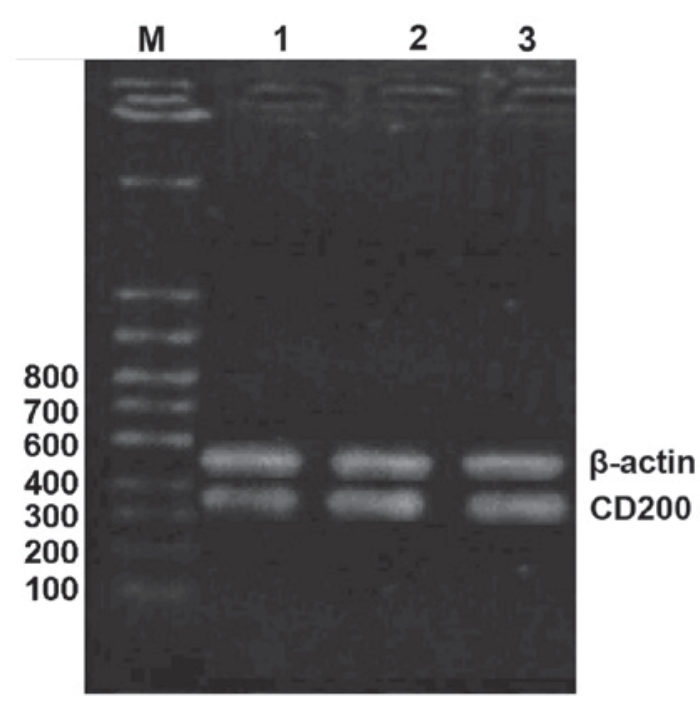

Figure 2. Result of detection by RT-PCR method. Lane 1, in active phase; lane 2, in stable phase; and lane 3, the control group.

among groups was tested (corrected) by $\chi^{2}$, ranked data were tested by rank sum; $\mathrm{P}<0.05$ was considered to indicate a statistically significant difference.

\section{Results}

Results of flow cytometry. The ratio of expression of CD200 on $\mathrm{CD}^{+} \mathrm{T}$ lymphocytes and $\mathrm{CD} 19^{+} \mathrm{B}$ lymphocytes of patients in active phase was significantly lower than those of patients in stable phase and control group $(\mathrm{P}<0.05)$. the ratio of expression of $\mathrm{CD}^{200}{ }^{+}$on $\mathrm{T}$ lymphocytes of patients in stable phase was significantly lower than that of the control group $(\mathrm{P}<0.05)$. The ratio of expression on $\mathrm{B}$ lymphocytes was compared with that of control group and the difference was not statistically significant (Fig. 1 and Table I). 

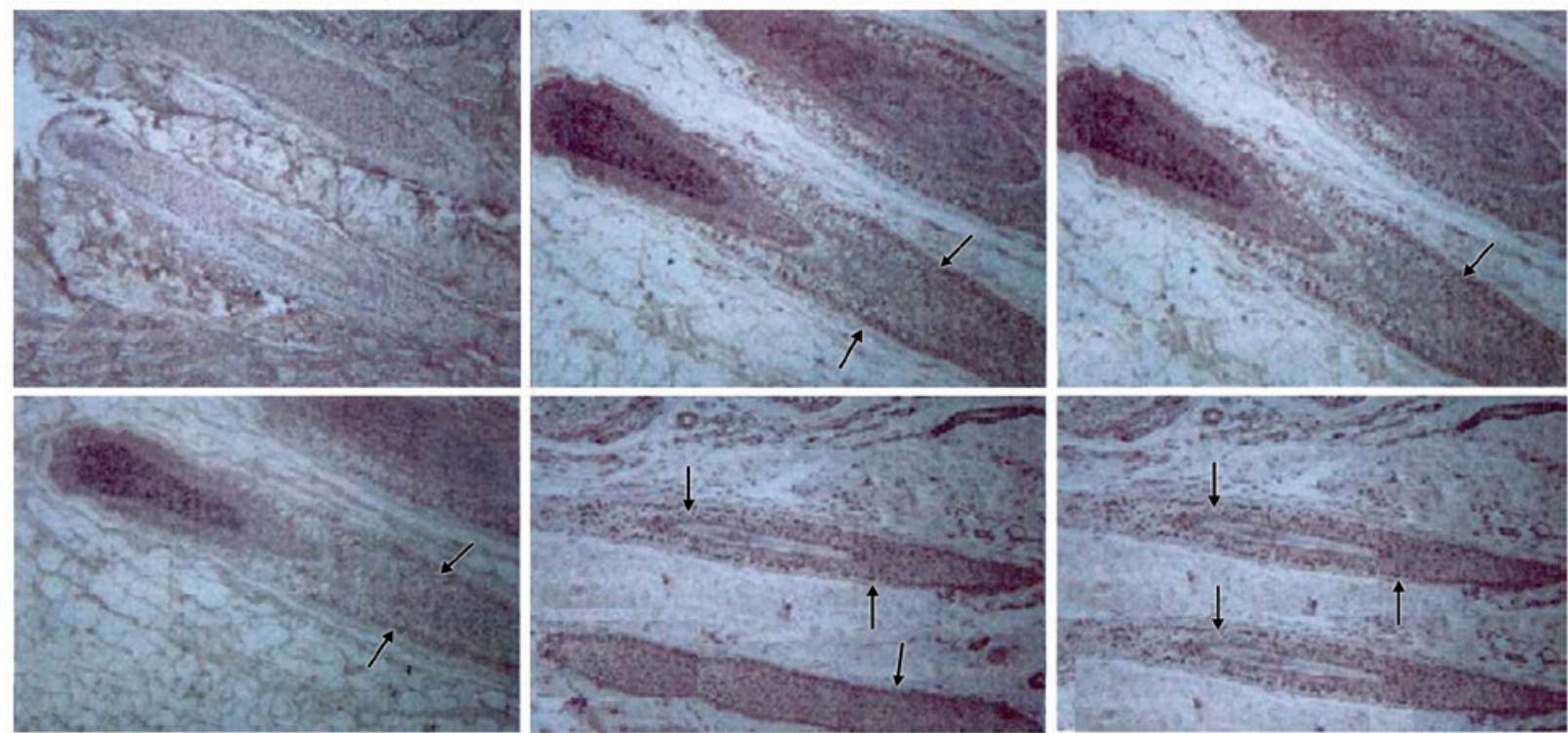

Figure 3. Results of immunohistochemical staining. The upper row CD200 and the bottom row CK15. The left is in active phase, the middle in stable phase, the right is the control group.

Table II. Results of immunohistochemical staining.

\begin{tabular}{|c|c|c|c|c|c|c|c|c|c|}
\hline \multirow[b]{2}{*}{ Groups } & \multirow[b]{2}{*}{ Cases } & \multicolumn{4}{|c|}{ CD200 } & \multicolumn{4}{|c|}{ CK15 } \\
\hline & & $\begin{array}{l}\text { Weakly } \\
\text { positive }\end{array}$ & $\begin{array}{l}\text { Moderate } \\
\text { positive }\end{array}$ & $\begin{array}{l}\text { Strong } \\
\text { positive }\end{array}$ & $\begin{array}{l}\text { Positive } \\
\text { rate }\end{array}$ & $\begin{array}{l}\text { Weakly } \\
\text { positive }\end{array}$ & $\begin{array}{c}\text { Moderate } \\
\text { positive }\end{array}$ & $\begin{array}{l}\text { Strong } \\
\text { positive }\end{array}$ & $\begin{array}{l}\text { Positive } \\
\text { rate }\end{array}$ \\
\hline Group in active phase & 50 & 10 & 9 & 6 & $25(50.0)$ & 5 & 9 & 30 & $44(88.0)$ \\
\hline Group in stable phase & 50 & 4 & 16 & 25 & $45(90.0)$ & 4 & 10 & 32 & $46(92.0)$ \\
\hline Control group & 50 & 3 & 14 & 30 & $47(94.0)$ & 3 & 12 & 33 & $48(96.0)$ \\
\hline$\chi^{2}$ & & & 49.738 & & 34.499 & & 3.193 & & 2.267 \\
\hline P-value & & & $<0.001$ & & $<0.001$ & & 0.784 & & 0.322 \\
\hline
\end{tabular}

Table III. Results of ELISA detection.

\begin{tabular}{lcc}
\hline Groups & IFN- $\gamma$ & IL-10 \\
\hline Group in active phase & $45.6 \pm 7.2$ & $10.8 \pm 4.2$ \\
Group in stable group & $37.2 \pm 5.9$ & $18.9 \pm 4.5$ \\
Control group & $35.4 \pm 5.3$ & $21.2 \pm 4.7$ \\
F-value & 8.264 & 7.528 \\
P-value & $<0.001$ & $<0.001$
\end{tabular}

ELISA, enzyme-linked immunosorbent assay; IFN- $\gamma$, interferon- $\gamma$; IL-10, interleukin-10.

Result of detection by RT-PCR method. The level of expression of CD200 mRNA in active phase was very significantly lower than those of the other two groups, the difference was statistically different $0.21 \pm 0.06$ to $0.43 \pm 0.08,0.49 \pm 0.06, F=9.425$, $\mathrm{P}<0.001$ (Fig. 2).

Result of immunohistochemical staining. The rate of positive expression of CD200 on the outer root sheath in active phase was significantly $(\mathrm{P}<0.05)$ lower than those of the other two groups. The moderately positive and strongly positive rates in active phase were significantly lower than those of the other two groups. The positive rates of CK were compared, the difference was not statistically significant (Fig. 3 and Table II).

Result of ELISA detection. The level of IFN- $\gamma$ increased significantly $(\mathrm{P}<0.05)$ in active phase, while the IL-10 level decrease was non-significant (Table III).

\section{Discussion}

Various studies have confirmed that $(1,6)$, alopecia areata is an autoimmune disease caused by hyperfunction of a specific Thl cell in the hair follicle. In the hair growth period, there are a large number of $\mathrm{T}$ lymphocytes infiltrating into the hair follicle and its surrounding alocepia area. The main tissue compatibility antigen MHC-I, II molecules in the area of alopecia areata express positive and normal tissue express negative. The expression of Th1/Th2 cytokines in the skin lesions and blood of patients with alopecia areata is out of balance and Th1 cytokines IFN- $\gamma$, IL-12 and TNF- $\alpha$ increase, while the Th2 
cytokines IL-4, -5, -10 decrease, and the change of the degree is positively associated with severity of the disease. IFN- $\gamma$ may play the leading role (7). Indeed, Gilhar et al (8) found that baldness can be induced in $\mathrm{C} 3 \mathrm{H} / \mathrm{HeJ}$ mice after intravenous injection with IFN- $\gamma$. They also found that hair can grow in the scalp of alopecia areata patients after being transplanted to immunodeficient mice subcutaneously, which suggests that the immune state of the host is relevant to the disease of alopecia areata.

At present, glucocorticoid is mainly used to treat SAA (9). CD200 belongs to type I membrane glycoprotein as a new member of the immunoglobulin super-family. It is widely distributed in human thymus cells, B cells, activated T cells, follicular dendritic cells, and vascular endothelial cells and in the plasma. It is also expressed in the central nervous system, testis, ovary and other immune organs (10) by the interaction with the receptor CD200R. It lowers the activity of myeloid cells, regulates immune response threshold value, regulates and controls cytokines to produce polarity (Th1/Th2 equilibrium), and participated in maintaining the immunity homeostasis of the body, specific tissues and organs (11). CD200 can be expressed with the hair follicle stem cell markers K14, 15 and 19, which makes it important in identifying and sorting hair follicle stem cells (12). Rosenblum et al transplanted CD200-deficient normal mouse fur grafts to normal mice with the same genus, which may lead to immune attack on hair follicles, resulting in hair loss of the skin grafting piece (13). They reported that CD200 can transfer 'no danger' signal for hair follicle and plays the role in maintaining hair follicle immune homeostasis. The level of IFN- $\gamma$ in the active phase significantly increased, and the level of IL-10 significantly decreased, suggesting that the decrease of CD200 expression may be related to the imbalance of Th1/Th2 balance.

In conclusion, CD200 expression level on peripheral blood and hair follicle outer root sheath of patients with SAA in active phase was decreased, which may be related to the imbalance of Th1/Th2.

\section{References}

1. Gilhar A and Kalish RS: Alopecia areata: a tissue specific autoimmune disease of the hair follicle. Autoimmun Rev 5: 64-69, 2006.

2. Paus R, Ito N, Takigawa M and Ito T: The hair follicle and immune privilege. J Investig Dermatol Symp Proc 8: 188-194, 2003.

3. Rosenblum MD, Olasz EB, Yancey KB, Woodliff JE, Lazarova Z, Gerber KA and Truitt RL: Expression of CD200 on epithelial cells of the murine hair follicle: a role in tissue-specific immune tolerance? J Invest Dermatol 123: 880-887, 2004.

4. Holmannová D, Kolácková M, Kondelková K, Kunes P, Krejsek J and Ctirad A: CD200/CD200R paired potent inhibitory molecules regulating immune and inflammatory responses; Part II: CD200/CD200R potential clinical applications. Acta Medica (Hradec Kralove) 55: 59-65, 2012.

5. Olsen EA, Hordinsky MK, Price VH, Roberts JL, Shapiro J, Canfield D, Duvic M, King LE Jr, McMichael AJ, Randall VA, et al; National Alopecia Areata Foundation: Alopecia areata investigational assessment guidelines - Part II. J Am Acad Dermatol 51: 440-447, 2004.

6. Ito T, Ito N, Bettermann A, Tokura Y, Takigawa M and Paus R: Collapse and restoration of MHC class-I-dependent immune privilege: exploiting the human hair follicle as a model. Am J Pathol 164: 623-634, 2004.

7. Freyschmidt-Paul P, McElwee KJ, Hoffmann R, Sundberg JP, Vitacolonna M, Kissling $\mathrm{S}$ and Zöller M: Interferon-gamma-deficient mice are resistant to the development of alopecia areata. Br J Dermatol 155: 515-521, 2006.

8. Gilhar A, Kam Y, Assy B and Kalish RS: Alopecia areata induced in $\mathrm{C} 3 \mathrm{H} / \mathrm{HeJ}$ mice by interferon-gamma: evidence for loss of immune privilege. J Invest Dermatol 124: 288-289, 2005.

9. Kim HS, Cho DH, Kim HJ, Lee JY, Cho BK and Park HJ: Immunoreactivity of corticotropin-releasing hormone, adrenocorticotropic hormone and alpha-melanocyte-stimulating hormone in alopecia areata. Exp Dermatol 15: 515-522, 2006.

10. Barclay AN, Wright GJ, Brooke G and Brown MH: CD200 and membrane protein interactions in the control of myeloid cells. Trends Immunol 23: 285-290, 2002.

11. Holmannová D, Kolácková M, Kondélková K, Kunes P, Krejsek J and Andrýs C: CD200/CD200R paired potent inhibitory molecules regulating immune and inflammatory responses; Part I: CD200/CD200R structure, activation, and function. Acta Medica (Hradec Kralove) 55: 12-17, 2012.

12. Ohyama M, Terunuma A, Tock CL, Radonovich MF, Pise-Masison CA, Hopping SB, Brady JN, Udey MC and Vogel JC: Characterization and isolation of stem cell-enriched human hair follicle bulge cells. J Clin Invest 116: 249-260, 2006.

13. Rosenblum MD, Yancey KB, Olasz EB and Truitt RL: CD200, a 'no danger' signal for hair follicles. J Dermatol Sci 41: $165-174,2006$. 